\title{
Appearance Control by Projector Camera Feedback for Visually Impaired
}

\author{
Toshiyuki Amano and Hirokazu Kato \\ Nara Institute of Science and Technology \\ 8916-5, Takayama, Ikoma, Nara 630-0192, Japan \\ \{amano,kato\}eis.naist.jp
}

\begin{abstract}
Visual impairment is a common problem for people worldwide. The projector-based AR technique has the ability to change the appearance of real objects, and it can help to improve visibility for the visually impaired. We propose a new framework for appearance enhancement with a projector camera system that employs a model predictive controller. This framework enables arbitrary image processing such as photo-retouch software in the real world and it helps to improve visibility for the visually impaired. In this article, we show the appearance enhancement results of Peli's method and Wolffshon's method for low vision, and Jefferson's method for color vision deficiencies. Through experimental results, the potential of our method to enhance the appearance for the visually impaired was confirmed to be the same as appearance enhancement for digital images and television viewing.
\end{abstract}

\section{Introduction}

Low vision and color vision deficiencies (CVD) are not minor visual impairments and both vision problems are improvable with projection-based augmented reality techniques in the real world. "Vision 2020: The Right To Sight" reports 153 million people are visually impaired by causes other than uncorrected refractive errors among the total of 314 million visually impaired people in the world[1]. The main cause of blindness and low vision was cataract except for uncorrected refractive errors, and many elderly people suffer these disabilities.

Peli et al.[13] proposed digital image enhancement techniques that enhance high-frequency components as aid for the visually impaired for central scotoma or cataracts. Wolffsohn et al. [16] examined the combination of generic edge detection and image processing to enhance the television viewing of the visually impaired. Both techniques are effective for improving visibility for low vision viewers and applicable to sight assistance in our life environment with real-time capture by video camera. However, users are tied down in front of the video monitor and it is not suitable for situations in daily life such as reading newspapers, books and looking at pictures.

The video see-through HMD has great capability for enhancement or annotation for visual impairment since it completely replaces human sight with small video displays attached in front of the eyes. However, to achieve a wide field of view, height resolution and high dynamic range display are still hard and these problems are frustrating to the user in daily use. From the aspect of assisting visual impairment, the optical see-through HMD is useful but precise registration that can overlay an edge line onto the real scene is still hard without strict support. The size and weight of both types of HMDs are becoming small and light but are still much more cumbersome than eye glasses.

Recently, several see-through optical processing devices have been developed and attempt to enhance the capabilities of the human vision system. Nayar et al.[12] proposed a human vision assist system that gives a user significant control over the radiometric and geometric properties by using a programmable imaging concept that uses a digital micro-mirror device (DMD) in the optical path. However, the resolution of the real scene for the user is restricted by the resolution of DMD since the real scene is observed via its reflection. Wetzstein et al.[15] proposed another way of see-through optical processing that used a Liquid Crystal Display (LCD) in the optical path call SOPhIE. The resolution of the real scene does not depend on the modulating device and it is easy to extend color image processing with the color LCD panel. These approaches have the ability to enhance the capability of the human vision system but have the same problems as HMD since these are definable as a kind of optical see-through HMD that uses a subtractive method of optics. Moreover, being tied down with special devices is not acceptable in terms of the quality of daily life.

In this paper, we propose a projection-based appearance enhancement technique for the visually impaired to achieve a better environment. The final style for the commercial product of a projector-based human vision system enhancement technique is supposable as a desk light or room light- 
ing, which means replacement of lighting systems currently in use. In our method, a projector camera dynamical feedback system was used. The system overlays a compensation pattern onto publications and gives the same appearance enhancement as Peli's and Wolffsohn's methods. This technique is applicable not only for low vision but for CVD as well. We focus attention on the fact that the apparent colors of publications are changeable with colored lighting and attempt visual improvement for the CVD viewer by projector camera dynamical feedback with Jefferson's method[11].

\section{Related Work and Contribution}

Recently, the projector-based AR technique has had two main trends of adapting and enhancing. For the adaptive method, projector camera feedback is proposed to control the appearance dynamically[9]. The closed-loop photometric adaptation[8] improved response speed for the appearance control and it enabled free positioning of a projector camera system with co-axial optics. For enhancing, the projector-based AR technique is also attempted for high dynamic display [4] and appearance enhancement [2]. These approaches are similar to photometric adaptation in terms of equipment configuration at a glance. However, these techniques have a new feature that enables enhancing the real environment optically, and it gives super-visibility that cannot be achieved with the poor quality of print media or limitations of the human vision system. The application of this technique is not only for print media, but also optical microscopes [3]. The dynamical feedback is also possible for appearance enhancement $[2,3]$ and this real-time processing makes sense for human vision support of visual impairment in daily life. Therefore, we used this real-time appearance enhancement for visual impairment but the techniques described in $[2,3]$ have a problem for visual impairment since those have no reference input. For the application for visual impairment, precise control is required and the reference input is necessary to achieve the desired appearance.

Our main contribution is adoption of reliable control theory for the projector camera system and enabling precise control for arbitrary image processing. Thanks to its versatility, conventional properties of appearance enhancement techniques are applicable, and it enables appearance enhancement for the visually impaired in the real world. In the follow section, we describe the adaptation of model prediction control for the projector camera feedback system.

\section{Projector Camera Feedback}

\subsection{Model Predictive Control}

The Dynamic Adaptation algorithm [8] can be explained as one of Model Predictive Control (MPC) [7], which has been commonly used in the processing industries such as

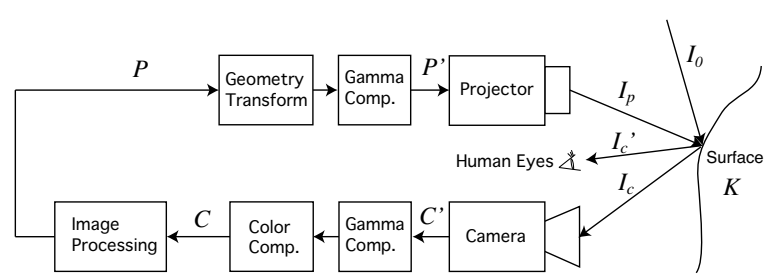

Figure 1. Previous System Diagram

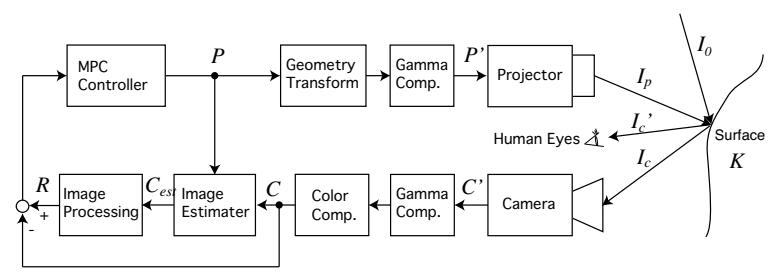

Figure 2. System Diagram with MPC controller

chemical plants and oil refineries since the 1980s. This control method is including a model for control object and manipulation value is decide by using of this model.

By using model prediction $y_{M}(t)$ that includes input $u(t)$ and measurement $y(t)$, estimation of output $y_{P}(t+1)$ is given by

$$
y_{P}(t+1)=y_{M}(t+1)+\operatorname{Error}(t)
$$

Where

$$
\operatorname{Error}(t)=y(t)-y_{M}(t)
$$

for considering of estimation error. In the MPC algorithm, reference trajectory

$$
y_{R}(t+1)=\alpha(t) y(t)+(1-\alpha(t)) r(t+1)
$$

is used for control stability against modeling error. Since $y_{P}(t+1)=y_{R}(t+1)$, we get control input for step $t+1$, where $\alpha(t)$ and $r(t)$ are tuning parameter and desired value for step $t$. The Dynamic Adaptation corresponds with this algorithm at the condition of $\alpha(t)=0$ and $\operatorname{Error}(t)=0$. Fortunately, projector camera feedback is stable without these terms in the case of irradiance compensation since control error is decreasing step-by-step. However, its error is critical for the appearance enhancement because it has a tendency of increasing estimation error in the case of enhancement.

\subsection{Negative Feedback for the Enhancement}

Amano et al. proposed an appearance enhancement technique with projector camera feedback [2]. The diagram is shown in Figure 1. In this diagram, all properties have three color components of $R, G$ and $B$ channels, and now we consider a single pixel relation. The environment light $I_{0} \in \mathcal{R}^{3}$ and projection light $I_{p} \in \mathcal{R}^{3}$ are mixed and reflected on the surface $K \in \mathcal{R}^{3 \times 3}$. This reflection

$$
I_{c}=K\left(I_{p}+I_{0}\right)
$$


is captured by camera and the system get an image

$$
C=M C_{c}^{\prime \gamma}, C^{\prime} \propto I_{c}
$$

via the gamma and color compensation. Regarding Lambert reflection, we can expect an irradiance observed by the user $I_{c}^{\prime} \approx I_{c}$, where $M$ is the color mixing matrix between camera and projector color spaces. After the image processing, the system projects compensation light $P$ with correct scaled irradiance onto the same point with the captured pixel from the projector by using the geometrical transformation and gamma compensation. The problem is that we cannot know what image processing is needed to achieve the final result because of many iterations in the feedback. Moreover, this feedback model is not robust since it does not include projection error or capture error.

To address these problems, we propose a new feedback approach shown in Fig. 2. By using a projection pattern, the estimated physical surface reflectance is written as

$$
\hat{K}=\operatorname{diag}\left\{C \cdot /\left\{\left(C_{\text {full }}-C_{0}\right) \odot P+C_{0}\right\}\right\} .
$$

where $C_{\text {full }}$ and $C_{0}$ are images captured under maximum power (white light) and minimum power (turn off) projections, ./ and $\odot$ mean component-wise division and multiplication respectively. Note, however, those non-linear response features of $C$ and $P$ are compensated with the module of "Gamma Comp." Based on $K$, the true appearance under the white light projection is given by

$$
C_{\text {est }}=\hat{K} C_{w h i t e},
$$

where $C_{w h i t e}=(1,1,1)^{T}$. Since this is a true appearance, we can get a reference image for the negative feedback loop via arbitrary image processing.

\subsection{MPC for Appearance Enhancement}

To apply the MPC algorithm, we use a projector camera response model

$$
C_{M}(t+1)=\hat{K}(t)\left\{\left(C_{\text {full }}-C_{0}\right) \odot P(t+1)+C_{0}\right\},
$$

where $P(t), C(t) \in([0,1],[0,1],[0,1])^{T}$ are normalized projection pattern and captured image at step $t$. Image prediction considering model error is written by

$$
C_{P}(t+1)=C_{M}(t+1)+\operatorname{Error}(t)
$$

where

$$
\operatorname{Error}(t)=C_{M}(t+1)+C(t)-C_{M}(t) .
$$

As for the reference trajectory, we used

$$
C_{R}(t+1)=\alpha C(t)+(1-\alpha) R(t+1)
$$

which employed a constant tuning parameter since projector camera response stays flat for $t \geq 0$, where $R(t+1)$ is a reference image given by the image processing result based on true appearance. From the control law $C_{P}(t+1)=$ $C_{R}(t+1)$, we get manipulating value

$$
\begin{aligned}
& P(t+1) \\
& \approx \hat{K}(t)^{-1}(1-\alpha)\{R(t+1)-C(t)\} \cdot /\left(C_{\text {full }}-C_{0}\right)+P(t)
\end{aligned}
$$

with an approximation of $\hat{K}(t) \approx \hat{K}(t-1)$. This approximation is employed to avoid near zero division when $\hat{K}(t-1) \approx 0$, which leads to unstable control. This approximation is tolerable since image prediction eq. (9) includes modeling error. The figure 3 shows the performance of proposed method by the examples of appearance control results of color saturation enhancement, color removal, color phase control, brightness equalization, and edge enhancement and blur.

\section{Appearance Enhancement for Low Vision}

Figure 4 shows the enhancement result with Peli's method[13]. The original appearance of the pictures shown in (a) is enhanced to (b) with projector camera feedback, and (c) shows the direct image processing result with Peli's method of (a). (d) (f) are intensity on the scenes expressed by spectrum. From these images, we can see the contrast boosted appearance at the edges of flower petals and a human face like the enhanced result shown on LCD. The difference of contrast is caused by the gamma curve of the LCD. If its strong contrast is helpful for low vision, it is applicable by a projector camera system by using contrast enhancement in reference image generation easily. For this enhancement, the projector (full-color XGA resolution) attached to a strong tripod is placed above the picture for the appearance enhancement and the camera (full-color VGA resolution) is attached to the projector. The geometrical and optical calibration for the projector camera system have been done previously with conventional methods. Its processing speed was 12.12 frames/sec with $2.4 \mathrm{GHz}$ cpu (Mac Book Pro Core2Duo 2.4GHz).

As with Peli's method, Wolffsohn's method [16] is applicable for our appearance enhancement technique since the proposed method employs a reference image. For the adaptation, we just implemented Wolffsohn's algorithm for the image processing part as with conventional static image processing. This versatility is one of the big benefits of our framework. Figure 5 shows the enhancement result by Wolffson's method. The edge of original appearance (a) is detected with a Prewitt filter and this result is overlaid with green color (b), the same as for the edge enhancement shown in [16]. This algorithm has been proposed for enhancement of television images to benefit the visually impaired but it can also assist in understanding the complex 


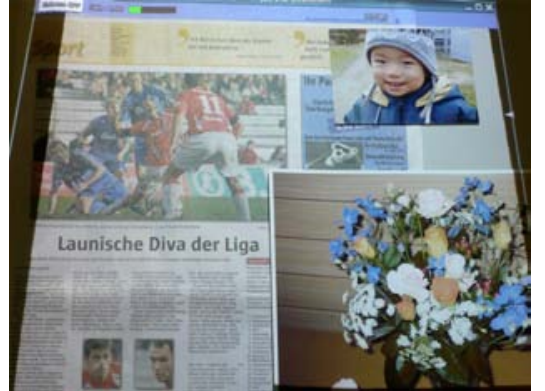

(a) Original Appearance

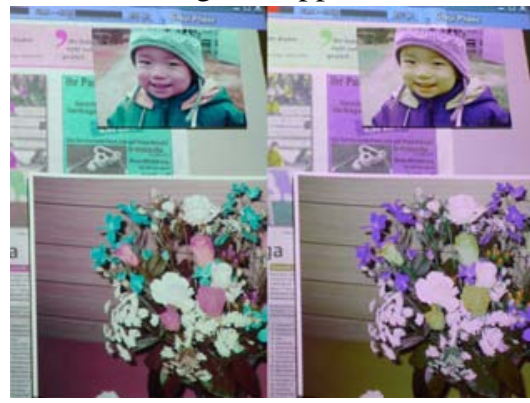

(d) Color Phase Control

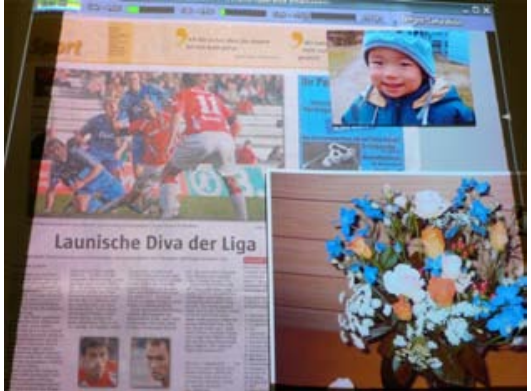

(b) Color Saturation Enhancement

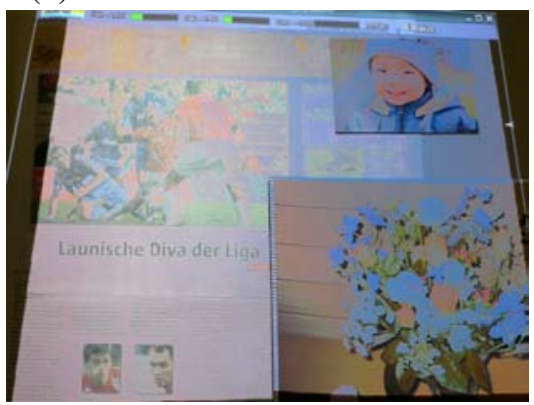

(e) Unique Brightness

Figure 3. Example of Appearance Control Results

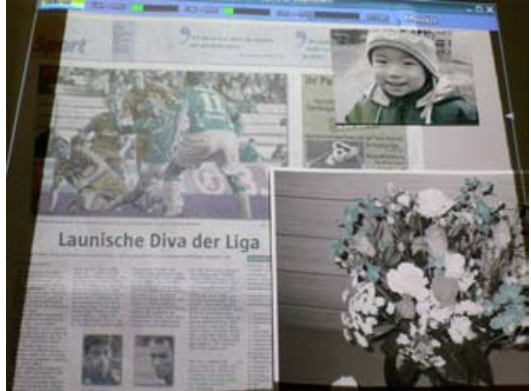

(c) Color Removal

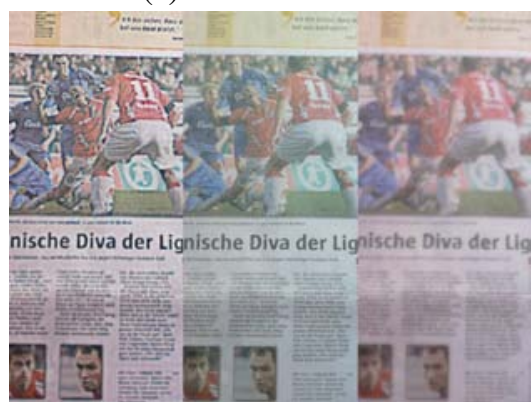

(f) Edge Enhance and Blur

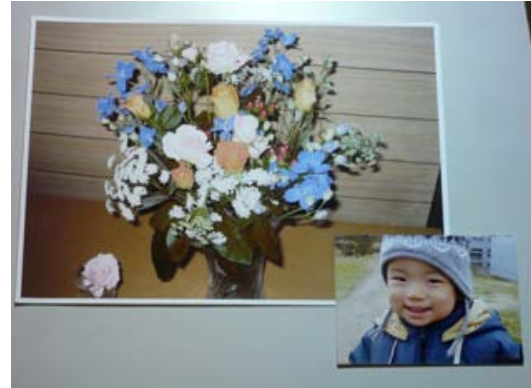

(a) The Orignal Appearance

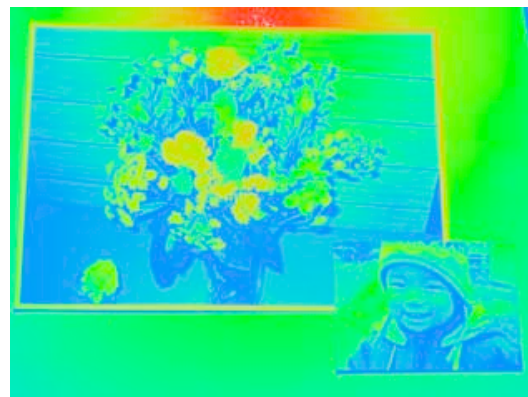

(d) Intensity of (a)

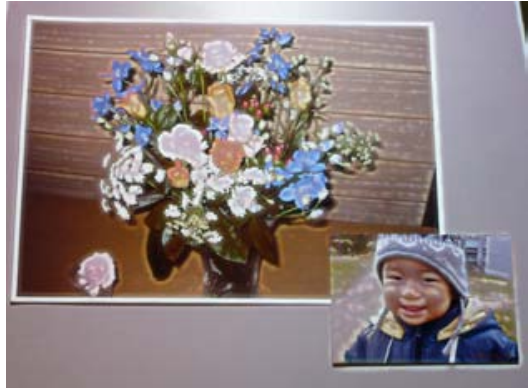

(b) Enhanced Result (Overlay Projection)

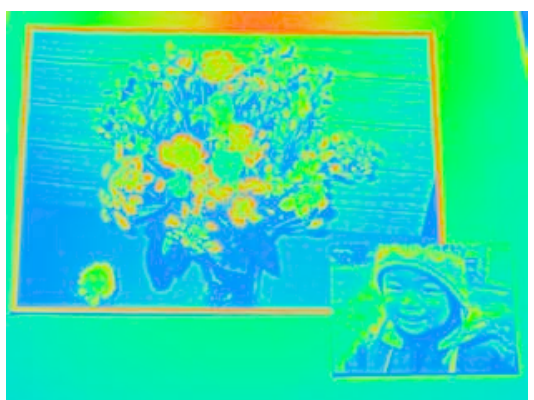

(e) Intensity of (b)

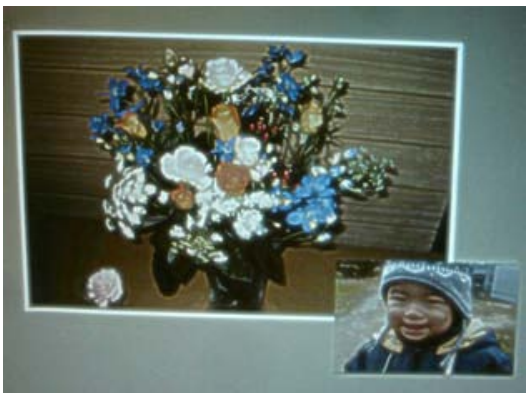

(c) Enhanced Result (LCD Display)

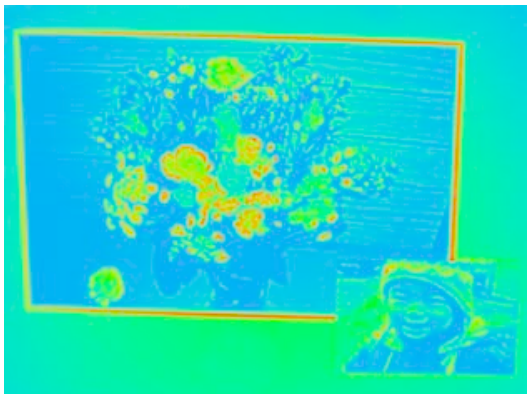

(f) Intensity of (c)

Figure 4. Enhancement Result by Peli's Method

scene in the printed picture. Figure 6 shows intensity on the scan line A-A' in Figure 5(a). Both results show the effectiveness of Wolffsohn's method, but it is obvious the resolution and sharpness are lost with the LCD. 


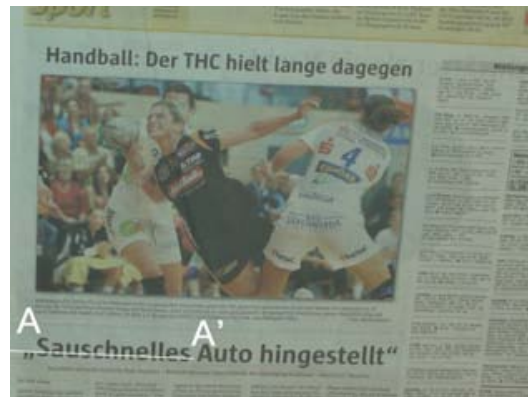

(a) The Orignal Appearance

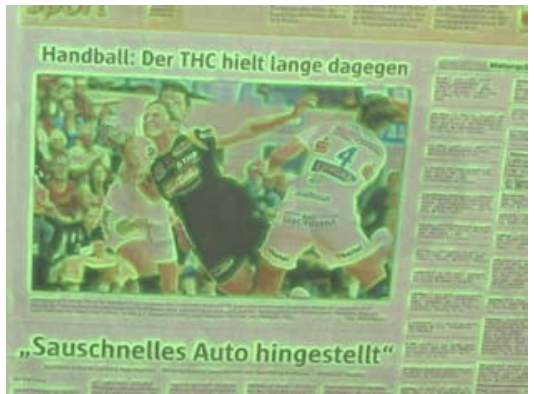

(b) Enhanced Result (Overlay Projection)

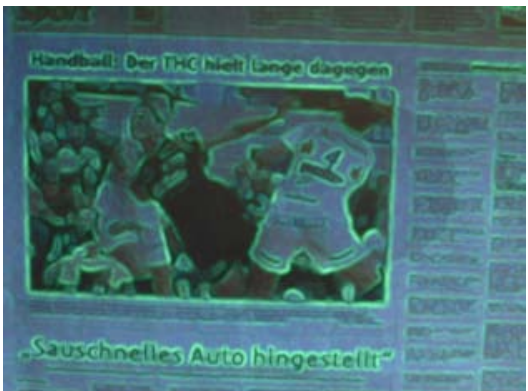

(c) Enhanced Result (LCD Display)

Figure 5. Enhancement Result by Wolffsohn's Method

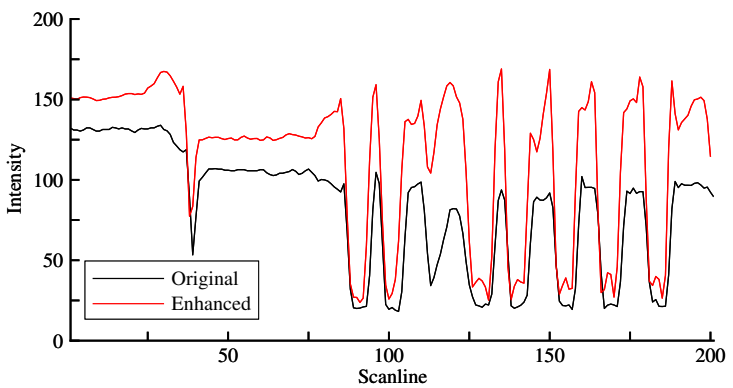

(a) Intensity on the real object (Overlay Projection)

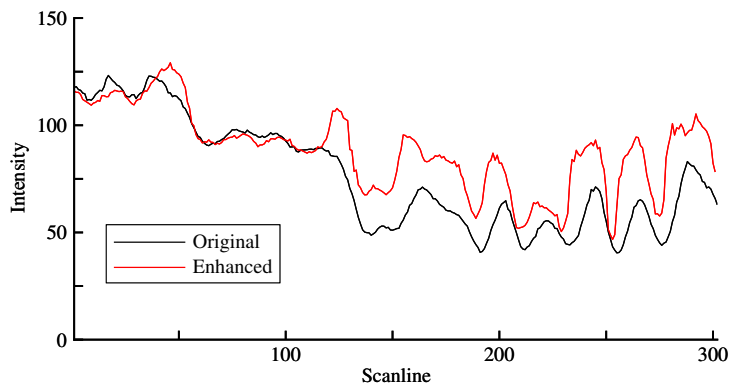

(b) Intensity on the LCD

Figure 6. Intensity at the Scan Line

\section{Color Management for CVD}

Several post-publication methods $[14,10]$ have been proposed to improve CVD accessibility. These methods are assumed to use a human interface for the CVD user and those methods replace color mapping. This replacement drastically improves visibility of the color graphs, figures and other graphical displays, but all of those methods are not suitable for time consumption. Moreover, projector-based color conversion has limits in terms of photometrics. The problem is we cannot convert a pure color object since it has no reflection except the limited spectrum band of an object's specific color band in theory. For these reasons, we applied Jefferson's method [11], which is simple linear color conversion that is used in the LMS color space based on color appearance simulation for dichromats [5]. This method does not make drastic color conversion but it can avoid extreme cases such as the Ishihara plate in publication with minimum color modification. It is good for viewing for natural scenes since it does not impart strange colors as with a blue-colored face.

Figure 7 shows the color enhancement result for the CVD. The appearance of Ishihara test plates is observed under a white light environment such as (a) with trichromatic perception. The numbers written on each plates are 7 (top left), 42 (top right), 57 (down left) and 8 (down right). However, these numbers cannot be seen with dichromatic perception (Protanope) except for the bottom right plate such as (b). This simulation is calculated from (a) with Vischeck [6]. It should be noted that the number in the bottom right plate is featly composed with two different colors and it is recognized with dichromatic perception. To use dichromatic perception simulation [5] for the reference input of projector camera feedback, we can show the appearance on the real world (c) such as a simulated image (b). This technique is useful for checking the accessibility of publishing for dichromatic perception. Jefferson's method modified the color of the original appearance of (d) for the trichromatic perception with the parameter $A_{P}=[0.0,-1.0,1.0,0.0]$, and its dichromatic perception is simulated like (e) with Vischeck. This parameter is effective for this case and it is chosen manually. The visibility is not better compared to trichromatic vision shown in (a). However, we can see correct numbers in (e) and it is effective to avoid extreme cases such as Ishihara test plates in daily life. This color modification is applicable for the other dichromatic perceptions with each transform matrix for deuteranopes and tritanopes.

The essential part of Jefferson's method is channel distribution expressed in the formula

$$
\tilde{C}_{L M S}=C_{L M S}+A_{i} \Delta C
$$




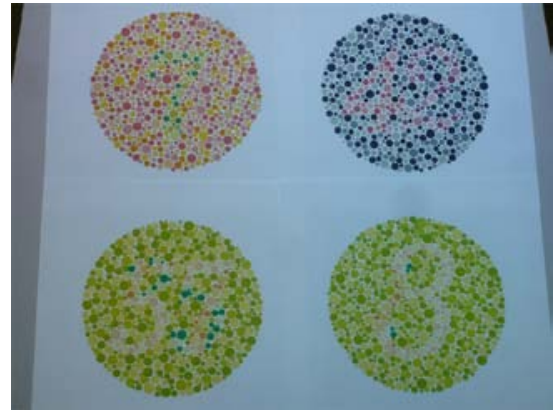

(a) Perception of trichromat

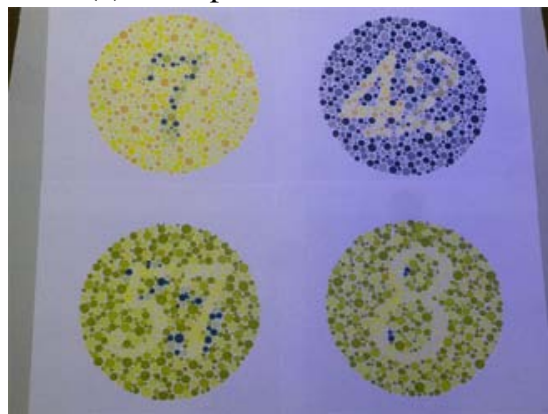

(d) Color modification for protanope

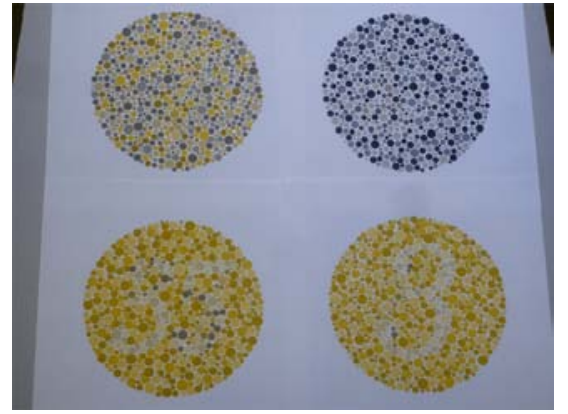

(b) Perception of dichromat

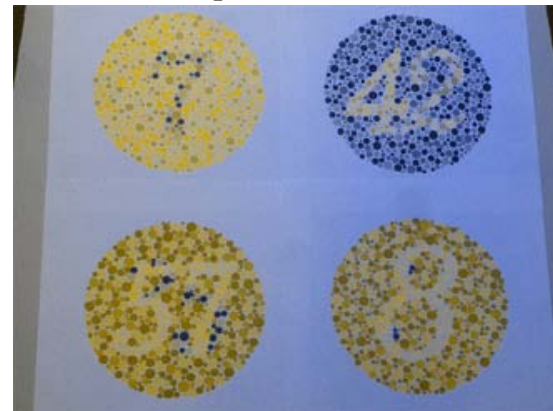

(e) Perception of (d) by dichromat Figure 7. Color Management Resuls

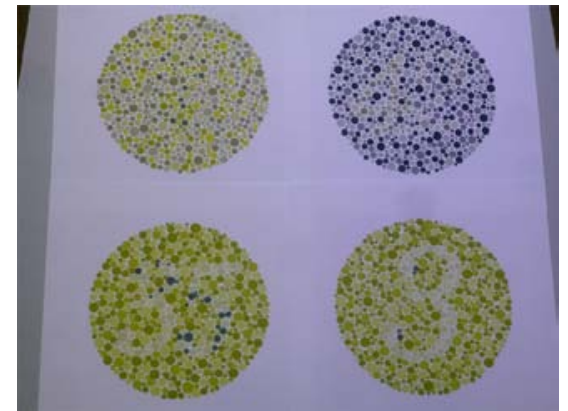

(c) Dichromatic simulation by overlay

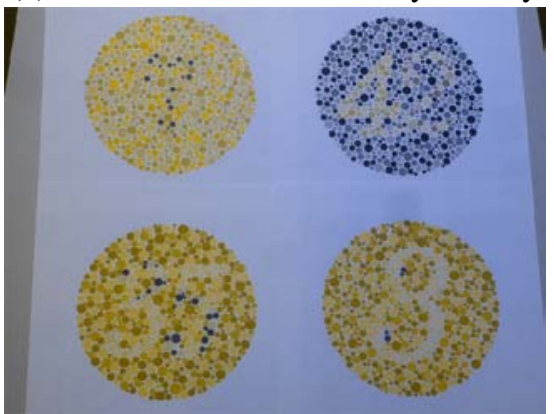

(f) Automatic tuning algorithm with matrix $A_{i}$. where $\tilde{C}_{L M S}$ is converted color, $C_{L M S}$ is original color and $\Delta C$ is the color difference between trichromatic and dichromatic perception on the LMS color space, and the index $i \in\{P, D, T\}$ denotes the type of dichromat. The diagonal elements of matrix $A_{i}$ are 1 for all types and the other elements are tuned with a smart user interface. However, we can understand there is no way other than to distribute $\Delta C$ to $M$ and $S$ channels for protanope since protanope cannot have an unobservable $L$ channel. Thus, the problem is how to distribute the $L$ component of $\Delta C$ to $M$ and $S$ channels. From this idea, we implemented the automatic tuning algorithm shown below for the protanope.

1. Extract foreground region $\mathcal{R}_{f}$ with background subtraction.

2. Calculate illuminance gravity $\bar{C}=\left(c_{l}, c_{m}, c_{s}\right)$ of original appearance in the region $\mathcal{R}_{f}$ with $L M S$ color space.

3. Calculate major direction $\theta_{P}=\tan ^{-1}\left(c_{s} / c_{m}\right)$.

4. Distribute the perception difference $\Delta C$ toward the orthogonal with major direction with the matrix $A_{P}=$ $\left[\begin{array}{ccc}1 & 0 & 0 \\ g \cos \left(\theta_{P}+\frac{\pi}{2}\right) & 1 & 0 \\ g \sin \left(\theta_{P}+\frac{\pi}{2}\right) & 0 & 1\end{array}\right]$.
From this algorithm, we got similar results as shown in Figure 7 (f) with (e).

\section{Conclusion}

In this article, we proposed a new framework for the projector camera system that employed model predictive control. This framework allows us to use conventional image enhancement techniques developed for visual impairment for the projector-based AR technique. For application to the visually impaired, we have shown the implementation of Peli's method and Wolffshon's method for low vision, and Jefferson's method for color vision deficiencies. The benefit of this framework is not only its ability with but also its configuration of equipment. Projector-based appearance enhancement is excellent in daily life since it is replaceable with a conventional lighting system. The other benefit is the cost of equipment. Conventional cameras and projectors are applicable to our framework and are inexpensive. Through experimental results, the potential of our method to enhance the appearance for the visually impaired was confirmed, but we still have problems for future work. The proposed framework works well for Jefferson's method, but it is impossible to change the appearance to the opposite color drastically for the pure-colored surface. This is caused by optical theory and impossible to solve with the current projector camera system. However, other suitable processing is able to consider color enhancement for projector camera 
system. We would like to find a more effective method for color vision deficiencies than Jefferson's method.

\section{References}

[1] VISION 2020 : The Right to Sight, Global initiative for the elimination of avoidable blindness: Action Plan 2006-2011. World Health Organization, 2007.

[2] T. Amano and H. Kato. Real world dynamic appearance enhancement with procam feedback. In Proc. of the 5th ACM/IEEE International Workshop on Projector camera systems, pages 1-2, 2008.

[3] O. Bimber, A. Grundhöfer, D. Kurz, S. Thiele, F. Häntsch, T. Amano, and D. Klöck. Projected light microscopy. In SIGGRAPH '09: SIGGRAPH 2009: Talks, pages 1-1, 2009.

[4] O. Bimber and D. Iwai. Superimposing dynamic range. In SIGGRAPH Asia '08: ACM SIGGRAPH Asia 2008 papers, pages 1-8, 2008.

[5] H. Brettel, F. Viénot, and J. D. Mollon. Computerized simulation of color appearance for dichromats. J. Opt. Soc. Am. A, 14(10):2647-2655, 1997.

[6] R. Dougherty and Wade. Vischeck: Vischeckurl, http://www.vischeck.com/vischeck/vischeckurl.php. accessed Mar. 23, 2010.

[7] J. E. N.-R. Eduardo F. Camacho, Carlos Bordons. Model predictive control. 1999.

[8] K. Fujii, M. D. Grossberg, and S. K. Nayar. A projectorcamera system with real-time photometric adaptation for dynamic environments. In Proc. of the Computer Vision and Pattern Recognition - Volume 2, page 1180, 2005.

[9] M. D. Grossberg, H. Peri, S. K. Nayar, and P. N. Belhumeur. Making one object look like another: Controlling appearance using a projector-camera system. Computer Vision and Pattern Recognition, IEEE Computer Society Conference on, 1:452-459, 2004.

[10] L. Jefferson and R. Harvey. Accommodating color blind computer users. In Assets '06: Proceedings of the 8th international ACM SIGACCESS conference on Computers and accessibility, pages 40-47, 2006.

[11] L. Jefferson and R. Harvey. An interface to support color blind computer users. In $\mathrm{CHI}$ '07: Proceedings of the SIGCHI conference on Human factors in computing systems, pages 1535-1538, 2007.

[12] S. K. Nayar, V. Branzoi, and T. E. Boult. Programmable imaging: Towards a flexible camera. Int. J. Comput. Vision, 70(1):7-22, 2006.

[13] E. Peli, E. Lee, C. L. Trempe, and S. Buzney. Image enhancement for the visually impaired: the effects of enhancement on face recognition. J. Opt. Soc. Am. A, 11(7):19291939, 1994.

[14] K. Wakita and K. Shimamura. Smartcolor: disambiguation framework for the colorblind. In Assets '05: Proceedings of the 7th international ACM SIGACCESS conference on Computers and accessibility, pages 158-165, 2005.

[15] G. Wetzstein, W. Heidrich, and D. Luebke. Optical Image Processing Using Light Modulation Displays. Computer Graphics Forum, 2009.
[16] J. S. Wolffsohn, D. Mukhopadhyay, and M. Rubinstein. Image enhancement of real-time television to benefit the visually impaired. American Journal of Ophthalmology, 144(3):436 - 440.e1, 2007. 